posibles explicaciones: errores de medición al calcular la edad gestacional y el número de visitas prenatales, y el hecho de que las posibles ventajas de la atención prenatal son menos pronunciadas a medida que mejora la salud reproductiva de una población. No obstante, los encargados de las políticas sanitarias deben seguir ocupándose de intensificar el cuidado prenatal de las muchas mujeres menos privilegiadas. (Goldani MZ, et al. Trends in prenatal care use and low birthweight in Southeast Brazil. Am J Public Health. 2004;94(8):1366-1371.)

\section{Régimen para reducir la tensión arterial}

Un nuevo informe reitera que la dieta Dietary Approaches to Stop Hypertension o conocida por su sigla DASH, puede llevar a una reducción notable de la tensión arterial. El régimen se basa en frutas, hortalizas, productos lácteos con grasa reducida, granos integrales, aves, pescado y nueces, y reducción de la ingesta de sodio. Este régimen se ha probado en dos ensayos en los que se comparó con la típica dieta estadounidense. En el primer estudio, la ingesta de sal se mantuvo a un promedio constante de $135 \mathrm{mmol}$ al día, es decir un poco menos que la ingesta promedio en los Estados Unidos. El segundo estudio fue un ensayo de eficacia ideado para evaluar los efectos principales e interactivos del régimen DASH y una ingesta reducida de sodio en la tensión arterial. Incluyó a 412 participantes con presión sistólica de 120 a 159 mmHg y diastólica de 80 a $95 \mathrm{mmHg}$. Después de dos primeras semanas consecutivas durante las cuales los participantes se alimentaron con una dieta de referencia de contenido sódico alto, se asignaron aleatoriamente a dos grupos: uno alimentado con el régimen DASH y el otro con una típica dieta estadounidense. Según el diseño cruzado, cada grupo fue expuesto consecutivamente a tres diferentes ingestas de sodio de 30 días cada una, en orden aleatorio. Las tres concentraciones de sodio (alta, intermedia y baja) tenían como objetivos 150, 100 o $50 \mathrm{mmol}$ al día (en la dieta de $2100 \mathrm{kcal}$ ). El contenido de sodio más elevado, típico del consumo estadounidense, era 50\% más alto que el intermedio, mientras que este representaba el límite superior de lo que se recomienda actualmente en el país.

La tensión arterial se midió tres veces antes de la inscripción, dos veces durante el período inicial de dos semanas y dos veces al día durante cinco de los últimos nueve días de cada período de alimentación de 30 días. Cada día se calculaba el promedio de las dos mediciones. Se consideró tensión arterial de inicio el promedio de los cinco pares de valores medidos antes de empezar las dietas asignadas. Los valores finales se obtuvieron del promedio de los cinco pares de mediciones al final de cada período de alimentación de 30 días. Se denominó hipertensión una presión sistólica $\geq 140 \mathrm{mmHg}$ o una diastólica $\geq 90 \mathrm{mmHg}$.

Tanto la reducción de sodio como la dieta DASH produjeron disminuciones de tensión arterial estadísticamente significativas y de importancia clínica en subgrupos definidos inicialmente por su estado de hipertensión, raza u origen étnico, sexo y edad. El extenso análisis de esos ensayos, recientemente publicado, presenta los resultados en la tensión arterial de subgrupos considerados a priori de importancia clínica y de subgrupos mixtos, como edad y estado de hipertensión, raza u origen étnico y el estado de hipertensión y género y raza u origen étnico. Mientras más reducida fue la ingesta de sodio, más se redujo la tensión arterial. Este resultado se observó en todos los participantes, independientemente de su raza, origen étnico, sexo, edad o estado original de hipertensión. Si bien la tensión arterial disminuyó al pasar de una dieta con alto contenido de sodio a una de contenido moderado, la disminución fue aproximadamente dos veces más intensa al pasar de la de contenido moderado a la de contenido bajo. Así que para normalizar la tensión arterial mediante la reducción de la ingesta de sodio, mientras más baja esta, mejor. Los investigadores también recomiendan la dieta DASH de la edad mediana en adelante, por sus efectos beneficiosos cuando aumentan los riesgos de enfermedad cardiovascular. (Bray GA, et al. A further subgroup analysis of the effects of the DASH diet and three dietary sodium levels on blood pressure: results of the DASH-Sodium Trial. Am J Cardiol. 2004;94(2):222-227.)

\section{Efecto del consumo de alcohol y de tabaco sobre la calidad del semen en humanos}

Algunos investigadores han notificado que el consumo de tabaco tiene efectos adversos sobre la concentración, aspecto morfológico y motilidad de los espermatozoides en el semen del ser humano; otros, en cambio, no han detectado efecto alguno. Lo mismo se aplica a los estudios efectuados para determinar si hay o no una relación entre la calidad del semen y el consumo de alcohol. En algunos casos se trata de estudios in vitro o con animales, y en otros, de estudios realizados con muestras pequeñas o en pacientes con problemas de fertilidad. Recientemente un grupo de investigadores en Argentina ha explorado estas relaciones con una muestra de 3976 hombres que fueron divididos en las siguientes categorías: los que no fuman; los que fuman 20 cigarrillos al día o menos; los que fuman más de 20 cigarrillos al día; los que no ingieren be- 